\title{
Adult attention-deficit/hyperactivity disorder, risky substance use and substance use disorders: a follow-up study among young men
}

\author{
Estévez-Lamorte, Natalia ; Foster, Simon ; Eich-Höchli, Dominique ; Moggi, Franz ; Gmel, Gerhard ;
} Mohler-Kuo, Meichun

\begin{abstract}
We investigated whether adult attention-deficit/hyperactivity disorder (ADHD) predicts risky substance use and substance use disorders (SUDs), and its impact on the course of these problematic substance use patterns. Our sample included 4975 Swiss men (mean age $20 \pm 1.2$ years) who participated in the baseline and 15-month follow-up assessments of the Cohort Study on Substance Use Risk Factors. We examined: (1) the contribution of ADHD, as assessed at baseline, on the risky use of alcohol, nicotine and cannabis, and their corresponding use disorders (AUD, NUD, CUD) at follow-up; and (2) the association between ADHD and the course of outcomes (i.e., absence, initiation, maturing out, persistence) over 15 months. All analyses were adjusted for socio-demographics and co-morbidity. Men with ADHD were more likely to exhibit persistent risky alcohol and nicotine use, and to mature out of risky cannabis use. ADHD at baseline was positively linked to AUD and negatively to CUD at follow-up, but not to NUD. For all SUDs, ADHD had a positive association with use persistence and maturing out. Comparing these two trajectories revealed that early age of alcohol use initiation distinguished between persistence and maturing out of AUD, while the course of NUD and CUD was related to ADHD symptoms and SUD severity at baseline. Already in their early twenties, men with ADHD are especially likely to exhibit persistent problematic substance use patterns. Substance-specific prevention strategies, particularly implemented before early adulthood, may be crucial to reducing the development and persistence of pathological patterns in such individuals.
\end{abstract}

DOI: https://doi.org/10.1007/s00406-018-0958-3

Posted at the Zurich Open Repository and Archive, University of Zurich

ZORA URL: https://doi.org/10.5167/uzh-163000

Journal Article

Accepted Version

Originally published at:

Estévez-Lamorte, Natalia; Foster, Simon; Eich-Höchli, Dominique; Moggi, Franz; Gmel, Gerhard; MohlerKuo, Meichun (2019). Adult attention-deficit/hyperactivity disorder, risky substance use and substance use disorders: a follow-up study among young men. European Archives of Psychiatry and Clinical Neuroscience, 269(6):667-679.

DOI: https://doi.org/10.1007/s00406-018-0958-3 
Adult attention-deficit/hyperactivity disorder, risky substance use and substance use disorders:

A follow-up study among young men

N. Estévez-Lamorte 1, 2, 3, 4 , S. Foster 2, 3, 4, D. Eich-Höchli ${ }^{5}$, F. Moggi ${ }^{6}$, G. Gmel 7, 8, 9, 10, M. Mohler-Kuo 1,2,3,4

\section{Affiliations}

${ }^{1}$ La Source, School of Nursing Sciences, University of Applied Sciences and Arts, Western Switzerland,

Lausanne

${ }^{2}$ Department of Child and Adolescent Psychiatry and Psychotherapy, University Hospital of Psychiatry Zurich, University of Zurich, Zurich, Switzerland

${ }^{3}$ Swiss Research Institute for Public Health and Addiction, Zurich, Switzerland

${ }^{4}$ Epidemiology, Biostatistics and Prevention Institute, Zurich, Switzerland

${ }^{5}$ Psychiatric University Hospital Zurich, Zurich, Switzerland

${ }^{6}$ University Hospital of Psychiatry, University of Bern, Bern, Switzerland

${ }^{7}$ Alcohol Treatment Centre, Lausanne University Hospital CHUV, Lausanne, Switzerland

${ }^{8}$ Addiction Switzerland, Lausanne, Switzerland

${ }^{9}$ Centre for Addiction and Mental Health, Toronto, Ontario, Canada

${ }^{10}$ University of the West of England, Bristol, United Kingdom

\section{Correspondence to:}

Natalia Estévez

University of Applied Sciences and Arts Western Switzerland, la Source

Avenue Vinet 30, 1004 Lausanne, Switzerland

Phone: +41797349283

E-mail: natalia.estevezgomez@uzh.ch

\section{Addresses of co-authors}

${ }^{1}$ University of Applied Sciences and Arts Western Switzerland, la Source, Avenue Vinet 30, 1004 Lausanne, Switzerland 
${ }^{2}$ Department of Child and Adolescent Psychiatry, University of Zurich, Neumünsterallee 9, 8032 Zurich, Switzerland

${ }^{5}$ Psychiatric University Hospital Zurich, Lenggstrasse 31, 8032 Zurich, Switzerland

${ }^{6}$ University Hospital of Psychiatry, University of Bern, Bolligenstrasse 111, 3000 Bern 60, Switzerland

${ }^{7}$ Alcohol Treatment Centre, Lausanne University Hospital CHUV, Avenue Beaumont 21bis, Pavillon 2, 1011

Lausanne, Switzerland

${ }^{8}$ Addiction Switzerland P.O. Box 870, 1001 Lausanne, Switzerland

${ }^{9}$ Centre for Addiction and Mental Health, 250 College St, Toronto, Ontario, M5T 1R8, Canada

${ }^{10}$ University of the West of England, Frenchay Campus Coldharbour Lane, Bristol BS16 1QY, United Kingdom

\section{Acknowledgments}

We thank Joseph Studer, Charlotte Eidenbenz and Laura Werlen for their valuable input. This study has been supported by the Swiss National Science Foundation (grant number: 33CS30_139467) and by the Hartmann Müller-Foundation (grant number: 1708). 


\begin{abstract}
Aim: We investigated whether adult attention-deficit/hyperactivity disorder (ADHD) predicts risky substance use and substance use disorders (SUDs), and its impact on the course of these problematic substance use patterns.

Methods: Our sample included 4,975 Swiss men (mean age $20 \pm 1.2$ years) who participated in the baseline and 15-month follow-up assessments of the Cohort Study on Substance Use Risk Factors. We examined: 1) the contribution of ADHD, as assessed at baseline, on the risky use of alcohol, nicotine and cannabis, and their corresponding use disorders (AUD, NUD, CUD) at follow-up; and 2) the association between ADHD and the course of outcomes (i.e., absence, initiation, maturing out, persistence) over 15 months. All analyses were adjusted for socio-demographics and co-morbidity.
\end{abstract}

Results: Men with ADHD were more likely to exhibit persistent risky alcohol and nicotine use, and to mature out of risky cannabis use. ADHD at baseline was positively linked to AUD and negatively to CUD at follow-up, but not to NUD. For all SUDs, ADHD had a positive association with use persistence and maturing out. Comparing these two trajectories revealed that early age of alcohol use initiation distinguished between persistence and maturing out of AUD, while the course of NUD and CUD was related to ADHD symptoms and SUD severity at baseline.

Conclusions: Already in their early twenties, men with ADHD are especially likely to exhibit persistent problematic substance use patterns. Substance-specific prevention strategies, particularly implemented before early adulthood, may be crucial to reducing the development and persistence of pathological patterns in such individuals.

Key words: adult attention-deficit/hyperactivity disorder, risky substance use, substance use disorder, alcohol, nicotine, cannabis 


\section{Introduction}

Attention-deficit/hyperactivity disorder (ADHD) is a neuropsychiatric disorder, which often has its onset and is most frequent during childhood, but may persist into adulthood [1, 2]. Thus, it can affect mental health and functioning in several life domains throughout one's entire life span [2-5]. In particular, ADHD has often been associated with problematic substance use patterns, like risky substance use and substance use disorders (SUDs) [6-12]. For instance, ADHD has been linked to a higher rate of SUDs related to various licit and illicit substances, more severe addiction, and a negative course of pathology (e.g., persistent symptoms, higher rate of relapse) [13, 14]. Thus, ADHD is considered a risk factor for developing and maintaining substance use problems. However, longitudinal studies addressing the contribution of ADHD on risky substance use and SUDs have demonstrated inconsistent results. While some studies have failed to identify an association between ADHD and problematic use patterns, others have confirmed it for some, but not other substances $[10,11,15]$. Consequently, it remains unclear whether the presence of ADHD truly is predictive of problematic use patterns at all; and, if so, for which substances.

Several methodological limitations might contribute to this inconsistency. Most previous investigations might have been biased by their use of non-representative convenience samples (e.g., clinical samples). Additionally, subject samples often were small; as such, adjustments for socio-demographics and co-morbidity, especially for conduct problems and antisocial behaviour, were unattainable in many studies and could have been confounders [10, 11]. In other investigations in which potential confounders were adjusted for in the analyses, the study's power might have been insufficient to detect any significant contribution of ADHD on outcomes $[12,16]$. Furthermore, many existing investigations addressing the contribution of ADHD on SUDs often followed children only into adolescence, failing to assess outcomes in young adults, even though substance use problems have their onset predominantly during this period of life [17]. Thus, in some studies, the absence of significant results might merely be because the participants were too young to develop such disorders [10, 15].

The period between late adolescence and young adulthood is a vantage point from which to examine whether ADHD influences the development and course of problematic substance use patterns. During this critical stage of life, individuals must deal with a number of normative developmental tasks that are accompanied by major changes in every life domain [18]. Additionally, substance use and related problems often increase, especially in men [19, 20]. Given the disadvantages that subjects suffering from ADHD experience in many relevant life domains, they may have more difficulties facing the new challenges that arise during this critical period and may, therefore, be at higher risk of exhibiting risky substance use or developing SUDs than their peers [6]. Indeed, in a cross-sectional study on a large, representative sample of young Swiss men, those with ADHD, were 1.5-2 times more likely than 
those without to exhibit the risky use of licit and illicit substances, and up to two times more likely to report SUDs, independently of socio-demographics and co-morbidity [12]. These findings also are consistent with those reported for a representative sample of the U.S. adult population [21]. However, given the cross-sectional nature of these investigations, causal inferences could not be drawn from the observations. In a recent longitudinal study by Vogel and colleagues [22] that used data from the same cohort of Swiss men as in [12] and investigated changes in substance use (i.e., between baseline and 15-month follow-up), young men with ADHD were not only more likely to use alcohol, nicotine and cannabis at baseline than men without ADHD, their consumption remained stable over a period of 15 months. Furthermore, in this study, men with ADHD who did not already use illicit substances at baseline were more likely to initiate their consumption between baseline and follow-up than those without ADHD. However, the investigators focused primarily on substance use and did not investigate changes in more severe patterns such as risky substance use and SUDs. It, therefore, remains unclear whether these results are transferable to problematic substance use patterns.

The first aim of the present study was to examine whether ADHD at baseline predicts the risky use of particular substances (i.e., alcohol, nicotine and cannabis) and related SUDs in young adults followed for a period of 15 months. The second aim was to examine the association between ADHD and the course of these problematic use patterns, providing a more thorough investigation of the effects of ADHD on possible changes (i.e., initiation or maturing out of problematic patterns) or persistent patterns (i.e., absence or persistence of problematic patterns) over the investigation period. To this end, we used data from a large, population-based sample of young Swiss men who were surveyed twice, with an interval of 15 months between the assessments. We hypothesized that, in young men, ADHD would predict the risky use of alcohol, nicotine and cannabis, as well as of related SUDs over the course of observation. Additionally, we hypothesized that men suffering from ADHD would be more likely to report the initiation and persistence of risky use and SUDs. To our knowledge, this is the largest sample of young men evaluated, within a longitudinal, population-based study, for the influence of ADHD on problematic substance use outcomes

\section{Methods}

Data were drawn from the 'Cohort Study on Substance Use Risk Factors' (C-SURF), which has been longitudinally investigating a cohort of young Swiss men. The protocol was approved by the Ethics Committee for Clinical Research at Lausanne University Medical School (Protocol No 15/07).

Participants were recruited at three of the six army recruitment centres in Switzerland, covering 21 of the 26 Swiss cantons. In Switzerland, army recruitment is mandatory; therefore, all young men must attend a formal evaluation 
to determine their eligibility for military or civil service, versus no service. As there is no pre-selection to army conscription, a representative sample of young Swiss men was accessible for study participation. All aspects of the study and the men's decision to participate were entirely independent of the army. The centres were used exclusively to enrol participants. The current study used data from the baseline (data collection: September 2010 to March 2012) and first follow-up assessments, the latter conducted roughly 15 months after the baseline assessment (data collection: January 2012 to April 2013).

\section{Participants}

Of 7,563 conscripts who provided written consent to participate, 5,990 (79.2\%) completed the baseline questionnaire. Of these, 5,223 (87.2\%) also filled out the follow-up questionnaire. This study used data collected from those participants who completed both assessments. Additionally, 248 (4.7\%) conscripts were excluded due to missing data on predictor variables. Thus, the final sample consisted of 4,975 subjects (95.3\% of the follow-up sample). To avoid losing further data, subjects with missing values on certain outcomes were not excluded from analyses involving other outcomes. For the exact number of participants used for each outcome variable, see Table 1. An analysis of possible non-response biases among the cohort of interest has been published previously [23]. Results from this prior investigation suggest that our participants and non-participants differed with respect to certain substance use outcomes. However, the differences identified were small, statistically significant mainly due to the large sample. Thus, we believe that non-response biases might only slightly affect the findings reported here.

\section{Independent Variables}

\section{Adult ADHD}

ADHD over the past 12 months was assessed at the baseline assessment using the 6-item Adult ADHD Self-Report Scale Screener (ASRS-v1.1) [24, 25]. Summation scores were dichotomized into "no ADHD” (scores 0-13) and "ADHD” (scores 14-24). The cut-off score of 14 has been previously shown to be optimal for the classification of clinician-defined ADHD cases versus clinician-defined non-ADHD cases [25]. Participants were excluded when more than two items were left unanswered. Missing responses for those who failed to answer only one or two items were replaced using nearest-neighbour hot-deck imputations, applying a random recursive partitioning dissimilarity matrix, implemented within the RRP package [26] running in version 2.15 of the R software (R Team Core Development, 17). It should be noted that, although the ASRS can be used to screen for potential ADHD cases, it does not provide sufficient information to establish a formal clinical diagnosis, as per the DSM-IV. Thus, 
in the context of our study, the term ADHD refers to people who reported ADHD symptoms and probably suffer from this disorder.

\section{Adjustment variables}

Subjects were screened for anti-social personality disorder (ASPD) at baseline using the Mini International Neuropsychiatric Interview (MINI plus, [27]). Answers were dichotomized to indicate the absence or presence of each symptom and coded as "no ASPD” or “ASPD”. ASPD was defined as at least two symptoms reported before and three symptoms after the age of 15 . For participants who answered all but two or fewer items, the same imputation procedure was applied as for ADHD. Subjects who failed to answer three or more questions were excluded.

Major Depression (MD) was assessed at follow-up using the Major Depressive Inventory (ICD-10) - WHO-MDI $[28,29]$. Answers were dichotomized to indicate the absence or presence of each symptom, and coded as "no MD" or "MD". MD was defined as at least five MDI items reported, with either item 1 or item 2 required to be among those five items [28]. Scores were computed when at least eight questions were answered.

Socio-demographic variables included in the analyses were subject age, linguistic region, highest achieved education, and degree of financial autonomy, which were assessed at follow-up; and information about maternal education and family affluence, collected at baseline. Categories for these variables are indicated in Table 1.

\section{Outcome variables}

\section{Risky substance use}

Risky alcohol use was assessed based on questions about the usual quantity and frequency of alcohol consumption and the frequency of risky single-occasion drinking (RSOD; consuming at least six standard drinks on a single occasion, over the last 12 months). This variable was dichotomized into "no risky use" (never or only occasionally drinking alcohol) versus "risky use” (either RSOD at least monthly, or risky-volume drinking). Risky-volume drinking was defined as drinking at least 21 standard drinks per week. For detailed information about the assessment and coding of these variables, see Gmel et al. [30].

Risky nicotine use was dichotomized into "no risky use" (never or only occasionally smoking cigarettes) versus "risky use" (smoking at least one cigarette daily).

Risky cannabis use was dichotomized into "no risky use" (using cannabis at most once per week or not at all) versus "risky use" (using cannabis more than once weekly). The aforementioned variables were created for both the baseline and follow-up assessments. 
To examine the course of risky alcohol, nicotine and cannabis use, a variable with four categories was created for each substance separately: 1) "absence of risky use" (no risky use reported at either data collection point); 2) "maturing out of risky use" (risky use at baseline, but not at follow-up); 3) "initiation of risky use" (no risky use reported at baseline, but reported at follow-up); and 4) "persistence of risky use" (risky use reported at both data collection points).

SUD

Alcohol use disorder (AUD) over the past 12 months was assessed via questionnaires, as in Knight et al. [31], including the additional criterion of "craving". The questions were adapted from the Semi-Structured Assessment for the Genetics of Alcoholism (SSAGA) [32, 33]. In accordance with the DSM-5, AUD was defined as reporting at least two criteria [34]. From the responses, a binary variable was created ("no AUD” vs. "AUD”).

Nicotine use disorder (NUD) was assessed using the Fagerström Test for Nicotine Dependence (FTND) revised version; [35, 36]. Summation scores were dichotomized into "no NUD” (scores 0-3) and "NUD” (scores 4-10) [37].

Cannabis use disorder (CUD) was measured with the Cannabis Use Disorder Identification Test (CUDIT) revised version [38], modified according to Annaheim et al. [39]. Summation scores were dichotomized into "no CUD" (scores 0-7) and "CUD" (scores 8-40). All the above-mentioned dichotomized variables were generated at both data collection points.

To investigate the course of AUD, NUD and CUD, a variable with four categories was created for each substance separately: 1) "absence of SUD" (no SUD of interest reported at either data collection point); 2) "maturing out of SUD” (the SUD of interest reported at baseline, but not at follow-up); 3) "initiation of SUD” (the SUD of interest not reported at baseline, but reported at follow-up); and 4) "persistence of SUD” (the SUD of interest reported at both assessments).

\section{Statistical analyses}

Data analysis was performed in SPSS 21.0. Socio-demographic, co-morbidity, and substance use characteristics were compared between participants with and without ADHD using Pearson chi-square analysis. To examine whether ADHD at baseline contributes to risky substance use (for alcohol, nicotine and cannabis) and related SUDs (AUD, NUD, CUD) at follow-up, binomial logistic regression analyses were performed for each of the six outcomes of interest. Odd ratios (ORs) were calculated between ADHD at baseline and each substance use outcome at follow-up, adjusting for 1) the corresponding risky use variable (i.e., risky use of alcohol, nicotine or cannabis) or SUD variable (i.e., AUD, NUD or CUD) at baseline alone; and 2) the corresponding risky use variable 
(i.e., risky use of alcohol, nicotine or cannabis) or SUD variable (i.e., AUD, NUD or CUD) at baseline, sociodemographic characteristics and co-morbid disorders (i.e., ASPD and MD). The presence of associations between ADHD and the course of risky substance use and SUDs were identified by means of multinomial logistic regression, again using ADHD as a predictor. These analyses were performed: 1) unadjusted, and 2) adjusted for socio-demographic characteristics and co-morbid disorders (i.e., ASPD and MD).

To identify differences between men with ADHD who matured out and those who reported a persistent pattern over the investigation period, post hoc analyses were conducted for each of the three SUDs (i.e., AUD, NUD and CUD). Based on previous research, which indicated that the characteristics of ADHD, severity of SUD at baseline, and age of substance use initiation can influence the course of SUDs [21, 40-44], we tested whether the persisting and maturing-out groups differed with respect to 1$)$ age at first use (<16 vs. $\geq 16$ years); 2) characteristics of ADHD (severity: size of score; type of symptoms: scores for 1. inattention and 2. hyperactivity-impulsivity); and 3) severity of the SUD of interest (number of symptoms at baseline).

\section{Results}

Characteristics of participants with and without ADHD are compared in Tables 1 and 2.

A total of 201 men screened positive and 4774 negative for ADHD. Compared to men without ADHD, those who endorsed ADHD symptoms tended to be older and from the French-speaking regions. They also were more likely to have completed primary school or have a high school/university-level education. Furthermore, relative to conscripts without ADHD, men reporting ADHD symptoms were more likely to report partial or complete financial dependency and had mothers who had completed high school/university or primary school only. Compared to men without ADHD, those with the disorder were also more likely to report ASPD and MD. Men with and without ADHD did not differ with respect to family affluence (for details, see Table 1).

Relative to conscripts without ADHD, those with ADHD symptoms tended to report persistent risky use of alcohol, nicotine and cannabis. Men with ADHD also more often matured out of risky cannabis use than those without the disorder. Compared to men without ADHD, those reporting symptoms were also more likely to report persistent AUD, NUD and CUD and to have matured out of these SUDs (for details, see Table 2).

\section{Risky substance use}

Binomial logistic regression analyses revealed no significant associations between the presence of ADHD at baseline and risky substance use at follow-up for alcohol, nicotine or cannabis. This was true both after adjusting 
only for the corresponding risky substance use variable at baseline, and after adjusting for the corresponding variable at baseline, as well as for socio-demographic characteristics and co-morbid disorders (Table 3).

Multinomial logistic regression analyses, both unadjusted and adjusted for socio-demographics and co-morbidity, also identified no significant link between ADHD and maturing out of or initiating the use of licit substances in a risky manner at follow-up. However, relative to participants who did not report risky use at either time point (reference category), conscripts with ADHD were more likely to report persistent risky use of alcohol, nicotine, and cannabis than those without ADHD. Furthermore, more men with ADHD ceased risky cannabis consumption at follow-up (matured out) than those without the disorder. After adjusting for socio-demographics and comorbidity, ORs were reduced, but remained significantly elevated for persistent risky alcohol and nicotine use, and for discontinuing cannabis use, but not for persistent risky cannabis consumption (Table 4).

SUD

Binomial logistic regression analyses revealed a positive association between ADHD at baseline and AUD at follow-up, both after adjusting only for AUD at baseline, and after adjusting for AUD at baseline, as well as for socio-demographic characteristics and co-morbidity. Furthermore, an inverse relationship was detected between ADHD and CUD at follow-up after adjusting for CUD at baseline, socio-demographics and co-morbidity (Table $3)$.

Multinomial logistic regression analyses, unadjusted and adjusted for socio-demographics and co-morbidity, revealed a significant link between the presence of ADHD and reporting a persistent pattern of AUD, NUD and CUD, as well as between ADHD and maturing out at follow-up. Compared with conscripts who did not report SUDs (i.e., AUD, NUD or CUD) at baseline or follow-up (reference category), men with ADHD were more likely to report persistent AUD, NUD and CUD than those without. Also, more participants with ADHD had matured out of AUD, NUD and CUD at follow-up than men without this disorder (Table 4).

Post hoc analysis of the men with ADHD revealed that those who matured out and those who had persistent AUD did not differ, with respect to characteristics of ADHD or AUD at baseline. However, participants with ADHD who continued to suffer from AUD at follow-up more commonly reported an earlier age at first alcohol use (i.e., before 16 years $)\left(\mathrm{x}^{2}(1)=7.11, \mathrm{p}=.008\right)$. Individuals with ADHD who matured out of NUD and those who reported a persistent pattern did not differ in age at nicotine use initiation, but the latter had significantly higher scores for $\operatorname{ADHD}(\mathrm{t}(35.8)=-3.47, \mathrm{p}=.001)($ i.e., higher scores for inattention $(\mathrm{t}(34.7)=-3.50, \mathrm{p}=.001))$ and also for NUD at baseline $(\mathrm{t}(36)=-2.83, \mathrm{p}=.008)$ than those who matured out. Relative to men who matured out of CUD, those who had persistent CUD reported higher scores for CUD at baseline $(\mathrm{t}(38)=-2.27, \mathrm{p}=.029)$. Additionally, a trend 
was found for hyperactivity symptoms, with men who matured out reporting higher scores for hyperactivity than those with persistent CUD $(\mathrm{t}(38)=1.81, \mathrm{p}=.078)$. No significant differences were found for age at first cannabis use.

\section{Discussion}

The two main purposes of the present study were: 1) to investigate whether ADHD predicts the risky use of particular substances (i.e., alcohol, nicotine, and cannabis) and related SUDs in young men followed for a period of 15 months; and 2) to examine for possible links between ADHD and the course (i.e., absence, initiation, maturing out, persistence) of problematic use patterns (i.e., risky use and SUD related to alcohol, nicotine and cannabis) over time.

Contrary to our expectations, ADHD did not predict the risky use of alcohol or nicotine at follow-up, once we adjusted for the corresponding risky substance use variable at baseline. However, it is important to note that men with ADHD were more likely to already report risky consumption of these licit substances at baseline, and continued to exhibit these risky use patterns more often at follow-up than those without ADHD. Thus, by the time of the investigation (i.e., when they are in their early 20s), the men we studied with ADHD had already developed risky use patterns for licit substances that appeared to persist over time. Such a persistent pattern is problematic, because regular excessive use of such substances could potentially increase one's risk of developing SUDs [6]. Additionally, alcohol and nicotine use at young ages are associated with other health-risk behaviours, their negative consequences, and various other adverse physical and mental health outcomes [6, 45-50]. Thus, continuing to use these substances in a risky manner over longer periods of time could, in the long-term, considerably compromise a person's functioning and mental health. In particular, the impairments that adults with ADHD experience secondary to their symptoms could be exacerbated, causing further disadvantages in life domains that are already affected by this disorder (e.g., at work) [46]. Therefore, to prevent additional illness burden in subjects suffering from ADHD during later phases of life, further efforts should be undertaken to reduce regular risky use of both alcohol and nicotine in young adults.

Our finding that ADHD at baseline predicted alcohol use disorder (AUD) at follow-up is consistent with those of two previously reported meta-analyses $[10,11]$. Both included several prospective longitudinal studies and investigated subjects over a longer period of time than the present study, starting in childhood and following subjects into adolescence or even adulthood. During the short investigation period of our study, participants with ADHD did not develop an AUD more often than those without, but those who met the study criteria for an AUD at baseline continued to suffer from this disorder at follow-up (only $27.8 \%$ (30/108) matured out), while men 
without ADHD were more likely to recover from AUD (42.5\% (616/1451) matured out). Indeed, the results of multinomial regression analysis suggest that men with ADHD are more likely to continue to meet criteria for AUD over time than those without. Consistent with previously-published findings, this observation suggests that ADHD can have an adverse effect on the course of AUD, resulting in the condition being more likely to persist $[13,51$, 52]. Young men suffering from ADHD may experience more difficulties controlling their substance use [6, 12, 53], and may need more support to overcome their alcohol-related problems than peers without ADHD.

We also noted that ADHD was positively associated with maturing out, suggesting that at least some men with ADHD recover from AUD. To our knowledge, no previous studies have generated a similar observation. Therefore, we tried (post hoc) to identify characteristics that might help to explain the occurrence of the two different trajectories (i.e., maturing out and persistence of AUD) among participants with ADHD. Our analyses revealed that men with ADHD and a persistent AUD more often reported an earlier age at first alcohol use (i.e., before 16 years). This result is consistent with a recent longitudinal population-based study, in which individuals with childhood ADHD who had an AUD already in their teens also were more likely to satisfy criteria for AUD as adults, while those who did not were less likely to drink heavily or have an increased risk of AUD in adulthood [51]. Our results further suggest that men with ADHD who start alcohol use later may be more likely to recover from AUD. Age of initiation seems to be a target for prevention. Therefore, to avoid the development and persistence of AUD in young adults, preventive strategies and counselling aimed at preventing the initiation of alcohol use need to be implemented early in life (i.e., during childhood and adolescence). Furthermore, in young adults suffering from both ADHD and an AUD, efforts should be intensified to help them recover from this use disorder to avoid its long-term persistence.

ADHD assessed at baseline did not predict nicotine use disorder (NUD) at follow-up. This result stands in contrast to a large body of literature addressing the relationship between ADHD and NUD (e.g., [7, 11, 43, 54]). However, this discrepancy might be caused by adjusting for NUD assessed at baseline, a procedure that was not applied in previous studies. Additionally, compared to the present study, these investigations started assessments during childhood or adolescence and followed subjects over a much longer period of time (i.e., 15-33 years). It is, therefore, also possible that our sample might be too old and/or the investigation period too short to identify a significant effect of ADHD on NUD. Indeed, our participants with ADHD were more likely to already have a NUD than those without ADHD at baseline (“ADHD”: 19.6\%; "no ADHD”: 9.5\%). Furthermore, a significant association between the presence of ADHD and persistence of NUD was evident. These results suggest that ADHD is, in fact, associated with an increased risk of NUD that most probably, typically develops before someone reaches 20 years of age, and persists over time. The results are also consistent with those of previous studies, in which 
individuals with ADHD experienced greater difficulty quitting smoking [14]. Furthermore, nicotine consumption has been frequently linked to self-medication for ADHD symptoms, particularly for those related to inattention $[55,56]$, which may further promote its regular use and, thereby, the persistence of NUD.

We also uncovered a positive association between ADHD and maturing out of NUD. A post hoc comparison between men with ADHD who had a persistent NUD and those who matured out revealed a different pattern, as for AUD. Indeed, they did not differ with respect to age at initiation, but the former group reported significantly more ADHD symptoms, particularly inattention symptoms, and had higher scores for NUD at baseline than those who matured out. These findings confirm previous evidence that inattention symptoms are an important predictor of both the presence and severity of NUD $[43,54]$. Treatments and prevention strategies designed to reduce inattention symptoms may thus help to prevent the development and persistence of NUD in young adults.

ADHD at baseline did not predict risky cannabis use at follow-up. However, we found a positive link between ADHD and maturing out of both risky cannabis use, and cannabis use disorder (CUD). Furthermore, logistic regression analysis revealed a negative relationship between ADHD and CUD. This result can be explained by the higher number of men with ADHD who met criteria for CUD at baseline and matured out at follow-up (45\%; 106/332), while more men without ADHD continued to suffer from CUD (only 31.9\% (18/40) matured out). Although men with ADHD ceased problematic cannabis consumption more often, a considerable proportion of these subjects still exhibited a persistent CUD at follow-up. Furthermore, in the study by Vogel et al. [22] over an interval of 15 months, more men with ADHD initiated cannabis use than those without; and even though at the follow-up assessment they did not meet criteria for risky consumption or SUDs, they could develop such problematic patterns later in life.

Post hoc analysis revealed that men with ADHD who already had more severe CUD at baseline were more likely to show a persistent pattern. Thus, preventive strategies aiming to diminish symptoms of CUD in young adults may be crucial to preventing the persistence of this use disorder. Furthermore, a trend $(\mathrm{p}<.10)$ was found for hyperactivity-impulsivity symptoms, in that men who matured out had higher scores for hyperactivity-impulsivity than those who had CUD at both assessments. Several studies have identified an association between greater hyperactivity symptoms and the development or presence of CUD [21, 57-60], which may also reflect either the difficulty these individuals have regulating their behaviour, or attempts to self-medicate hyperactivity-impulsivity symptoms. From our data, we can only speculate as to why individuals with higher scores were more likely to recover from CUD, though it is possible that, after ceasing their cannabis consumption, they turned towards using other substances to alleviate their symptoms. In fact, Vogel and colleagues [22] found that men with ADHD at baseline were at risk of initiating the consumption of other illicit drugs and non-medical prescription drugs at 15- 
month follow-up. Whether there is a reduction in both cannabis use and related disorders, or a substitution with other drugs, needs to be clarified in future investigations. This is pivotal, if we are to take appropriate measures to design interventions that can truly help adults suffering from ADHD and avoid further burdens during later stages of life.

This study has several limitations. First, women were excluded from our study, even though the associations we investigated may differ between men and women [40]. Second, ADHD, co-morbid disorders and SUDs were assessed by self-report and without including further confirmatory diagnostic assessments. However, all the instruments we used are well-validated scales, frequently utilized in previous research [25, 27-29, 32, 33, 35, 39]. Nevertheless, it is important to note that, with a cut-off of 14 points, the sensitivity of the ASRS Screener has been reported as only $64.9 \%$ [25]. This means that a considerable number of ADHD cases might have screened negative. We expect that these individuals had less severe symptoms, on average, than those who screened positive [24]. This is mainly because the ASRS version we used (version 1.1) and the clinical interviews applied for its validation were based upon DSM-IV criteria that were originally developed for children and are too conservative for adults $[61,62]$. For example, adults often exhibit fewer ADHD symptoms than children, but still experience significant functional impairment and mental health problems (e.g., SUDs). According to the DSM-IV definition, these individuals would fail to meet enough criteria for a clinical diagnosis of ADHD and would, therefore, be classified as non-cases. Because misclassified individuals with ADHD may also show risky substance use patterns or even suffer from SUDs, this misclassification may have biased our findings. Third, we did not assess subjects' medical history for any of the disorders of interest; thus, their true clinical status was unknown. Additionally, no information was collected about received or current treatments, which may have influenced whether men with ADHD matured out or continued to suffer from SUDs. Fourth, we used the ASRS Screener, which was designed to assess $\mathrm{ADHD}$ as a total score and not to differentiate between inattention and hyperactivity-impulsivity symptoms. Therefore, even though our post hoc evaluations were consistent with previous studies [21, 63], future studies need to address the influence of each symptom type on the course of SUDs more thoroughly, using instruments that are suitable for assessing inattention and hyperactivity-impulsivity symptoms separately. Fifth, the investigation interval of 15 months was relatively short, and the number of subjects whose substance use changed over this period (i.e., those who matured out of or developed an SUD at follow-up) was small for some substances, like nicotine. Therefore, whether these trajectories reflect a constant phenomenon and not just temporary changes, or are the result of bias, must be addressed in future studies using longer intervals between assessments. Indeed, our findings may have been biased by drop-outs or the exclusion of participants with missing values for predictor variables. Those who fulfilled criteria for NUD or CUD at baseline were more likely to drop- 
up at follow-up or be excluded (data not shown) and, thereby, not be included in our final analysis. Furthermore, this difference was more prominent among those without ADHD (NUD: men with ADHD, $\mathrm{x}^{2}=0.72, \mathrm{p}=.39$; men without ADHD, $x^{2}=68.58, p<.001$; CUD: men with ADHD, $x^{2}=3.04, p=.08$; men without $\mathrm{ADHD}, \mathrm{x}^{2}=45.73$, $\mathrm{p}<.001$ ), and this imbalance between the two groups may have biased the results. Finally, it must be noted that the observation that men with ADHD do mature out of AUD, NUD and CUD more often than those without ADHD is unexpected and cannot be explained on the basis of previous findings. One possible explanation is that people with ADHD may have been more likely to attend regular therapy sessions with a psychiatrist than those without ADHD, where mention of their substance problems could have led either to appropriate therapy or to selfdiscovery of strategies that they could then use to control their behaviours. However, it is also possible that the maturing out we observed might only reflect a change in their primary substance of use [22]. To shed light on this particular trajectory, further investigations that incorporate detailed medical and treatment histories are required.

\section{Conclusions}

In conclusion, over a relatively brief time window of 15 months, we found that ADHD was linked to the persistence of risky alcohol and nicotine consumption, but also to the maturing out of risky cannabis use. These findings are somewhat consistent with those observed for more severe consumption patterns (i.e., related SUDs). In fact, ADHD was identified as a risk factor for AUD and was negatively associated with CUD. Examination of the courses of AUD, NUD and CUD revealed that, for these disorders, ADHD is essentially associated with two trajectories: 1) persistence, and 2) maturing out of problematic use. Identifying characteristics that distinguish between adults exhibiting persistence versus maturing out could help to detect subjects who are at risk of ultimately developing addictions, and might aid in designing appropriate prevention strategies that better fit the needs of young men with ADHD. Our evaluation also suggests that the relevance of certain characteristics may differ, depending on the substance. Thus, substance-specific preventive interventions may be central to reducing the risk and persistence of SUDs. Finally, our findings indicate that, as early as their early 20s, men with ADHD are more likely to have problematic substance use. Moreover, though some mature out of this use over time, for most these patterns appear to persist over time, suggesting that, by this age, the damage may be already done. Preventive strategies may, therefore, be more successful when administered before early adulthood. Given the short observation period in the present study, our results, particularly those related to maturing out, need to be corroborated in future follow-up studies with a longer interval between assessments.

\section{Ethical standards}


The authors assert that all procedures contributing to this work comply with the ethical standards of the relevant national and institutional committees on human experimentation and have, therefore, been performed in accordance with the Declaration of Helsinki 1964 and its later amendments. All participants gave their informed consent prior to their inclusion in the study.

\section{Conflicts of interest}

On behalf of all authors, the corresponding author states that there are no conflicts of interest.

\section{References}

1. Faraone S V, Biederman J, Spencer T, et al. (2000) Attention-deficit/hyperactivity disorder in adults: an overview. Biol Psychiatry 48:9-20. doi: 10.1016/S0006-3223(00)00889-1

2. Ebejer JL, Medland SE, van der Werf J, et al. (2012) Attention Deficit Hyperactivity Disorder in Australian Adults: Prevalence, Persistence, Conduct Problems and Disadvantage. PLoS One. doi: 10.1371/journal.pone.0047404

3. Brod M, Schmitt E, Goodwin M, et al. (2012) ADHD burden of illness in older adults: A life course perspective. Qual Life Res 21:795-799. doi: 10.1007/s11136-011-9981-9

4. Das D, Cherbuin N, Butterworth P, et al. (2012) A population-based study of attention deficit/hyperactivity disorder symptoms and associated impairment in middle-aged adults. PLoS One 7:1-9. doi: 10.1371/journal.pone.0031500

5. De Zwaan M, Gruß B, Müller A, et al. (2012) The estimated prevalence and correlates of adult ADHD in a German community sample. Eur Arch Psychiatry Clin Neurosci 262:79-86. doi: 10.1007/s00406-011-02119

6. Baker L, Prevatt F, Proctor B (2012) Drug and Alcohol Use in College Students With and Without ADHD. J Atten Disord 16:255-263. doi: 10.1177/1087054711416314

7. Klein RG (2012) Clinical and Functional Outcome of Childhood Attention-Deficit/Hyperactivity Disorder 33 Years Later<alt-title>Outcome of Childhood ADHD 33 Years Later</alt-title>. Arch Gen Psychiatry 69:1. doi: 10.1001/archgenpsychiatry.2012.271

8. Sibley MH, Pelham WE, Molina BSG, et al. (2012) When Diagnosing ADHD in Young Adults Emphasize Informant Reports, DSM Items, and Impairment. J Consult Clin Psychol 80:1052-1061. doi: $10.1037 / \mathrm{a} 0029098$

9. Sibley MH, Pelham WE, Molina BSG, et al. (2012) Diagnosing ADHD in adolescence. J Consult Clin Psychol 
80:139-150. doi: 10.1037/a0026577

10. Charach A, Yeung E, Climans T, Lillie E (2011) Childhood attention-deficit/hyperactivity disorder and future substance use disorders: Comparative meta-analyses. J Am Acad Child Adolesc Psychiatry 50:9-21. doi: 10.1016/j.jaac.2010.09.019

11. Lee SS, Humphreys KL, Flory K, et al. (2011) Prospective association of childhood attentiondeficit/hyperactivity disorder (ADHD) and substance use and abuse/dependence: A meta-analytic review. Clin Psychol Rev 31:328-341. doi: 10.1016/j.cpr.2011.01.006

12. Estévez N, Dey M, Eich-Höchli D, et al. (2015) Adult attention-deficit/hyperactivity disorder and its association with substance use and substance use disorders in young men. Epidemiol Psychiatr Sci 1-12. doi: $10.1017 /$ S2045796015000360

13. Wilens TE, Upadhyaya HP (2007) Impact of substance use disorder on ADHD and its treatment. J Clin Psychiatry 68:e20.

14. Humfleet GL, Prochaska JJ, Mengis M, et al. (2005) Preliminary evidence of the association between the history of childhood attention-deficit/hyperactivity disorder and smoking treatment failure. Nicotine Tob Res 7:453-460. doi: 10.1080/14622200500125310

15. Galéra C, Pingault J-B, Fombonne E, et al. (2013) Attention problems in childhood and adult substance use. J Pediatr 163:1677-1683.e1. doi: 10.1016/j.jpeds.2013.07.008

16. Serra-Pinheiro M a., Coutinho ESF, Souza IS, et al. (2012) Is ADHD a Risk Factor Independent of Conduct Disorder for Illicit Substance Use? A Meta-Analysis and Metaregression Investigation. J Atten Disord. doi: $10.1177 / 1087054711435362$

17. Kessler RC, Berglund P, Demler O, et al. (2005) Lifetime Prevalence and Age-of-Onset Distributions of. Arch Gen Psychiatry 62:593-602. doi: 10.1001/archpsyc.62.6.593

18. Gotham HJ, Sher KJ, Wood PK (2003) Alcohol involvement and developmental task completion during young adulthood. J Stud Alcohol 64:32-42.

19. Rehm J, Taylor B, Room R (2006) Global burden of disease from alcohol, illicit drugs and tobacco. Drug Alcohol Rev 25:503-513. doi: 10.1080/09595230600944453

20. Toumbourou J, Stockwell T, Neighbors C, et al. (2007) Interventions to reduce harm associated with adolescent substance use. Lancet 369:1391-1401. doi: 10.1016/S0140-6736(07)60369-9

21. De Alwis D, Lynskey MT, Reiersen AM, Agrawal A (2014) Attention-deficit/hyperactivity disorder subtypes and substance use and use disorders in NESARC. Addict Behav 39:1278-85. doi: 10.1016/j.addbeh.2014.04.003 
22. Vogel T, Dom G, van de Glind G, et al. (2016) Is attention deficit/hyperactivity disorder among men associated with initiation or escalation of substance use at 15-month follow-up? A longitudinal study involving young Swiss men. Addiction 111:1867-1878. doi: 10.1111/add.13422

23. Studer J, Baggio S, Mohler-Kuo M, et al. (2013) Examining non-response bias in substance use research-Are late respondents proxies for non-respondents? Drug Alcohol Depend 132:316-323. doi: 10.1016/j.drugalcdep.2013.02.029

24. Kessler RC, Adler L, Ames M, et al. (2005) The World Health Organization Adult ADHD Self-Report Scale (ASRS): a short screening scale for use in the general population. Psychol Med 35:245-256. doi: $10.1017 / \mathrm{S} 0033291704002892$

25. Kessler RC, Adler L a., Gruber MJ, et al. (2007) Validity of the World Health Organization Adult ADHD SelfReport Scale (ASRS) Screener in a representative sample og health plan members. Int J Methods Psychiatr Res 16:52-65. doi: 10.1002/mpr.208

26. Iacus SM, Porro G (2007) Missing data imputation, matching and other applications of random recursive partitioning. Comput Stat Data Anal 52:773-789. doi: 10.1016/j.csda.2006.12.036

27. Lecrubier Y, Weiller E, Hergueta T, et al. (1998) Mini mini international neuropsychiatric interview. French version 5.00. Hop. la pitié salpétriere, Inser. Paris, Fr.

28. Bech P, Rasmussen N a, Olsen LR, et al. (2001) The sensitivity and specificity of the Major Depression Inventory, using the Present State Examination as the index of diagnostic validity. J Affect Disord 66:159164. doi: 10.1016/S0165-0327(00)00309-8

29. Olsen LR, Jensen D V, Noerholm V, et al. (2003) The internal and external validity of the Major Depression Inventory in measuring severity of depressive states. Psychol Med 33:351-6.

30. Gmel G, Gaume J, Willi C, et al. (2010) Challenging the "inoffensiveness" of regular cannabis use by its associations with other current risky substance use - A census of 20-year-old Swiss men. Int J Environ Res Public Health 7:46-59. doi: 10.3390/ijerph7010046

31. Knight JR, Wechsler H, Kuo M, et al. (2002) Alcohol abuse and dependence among U.S. college students. J Stud Alcohol 63:263-70.

32. Bucholz KK, Cadoret R, Cloninger CR, et al. (1994) A new, semi-structured psychiatric interview for use in genetic linkage studies: a report on the reliability of the SSAGA. J Stud Alcohol 55:149-58.

33. Hesselbrock M, Easton C, Bucholz KK, et al. (1999) A validity study of the SSAGA--a comparison with the SCAN. Addiction 94:1361-70.

34. Hasin DS, O'Brien CP, Auriacombe M, et al. (2013) DSM-5 criteria for substance use disorders: 
recommendations and rationale. Am J Psychiatry 170:834-51. doi: 10.1176/appi.ajp.2013.12060782

35. Heatherton TF, Kozlowski LT, Frecker RC, Fagerström KO (1991) The Fagerström Test for Nicotine Dependence: a revision of the Fagerström Tolerance Questionnaire. Br J Addict 86:1119-27.

36. Bleich S, Havemann-Reinecke U, Kornhuber J (2002) Fagerström-test für nikotinabhängigkeit (ftna).

37. Huang CL, Lin HH, Wang HH (2008) Evaluating screening performances of the Fagerstrom tolerance questionnaire, the Fagerstrom test for nicotine dependence and the heavy smoking index among Taiwanese male smokers. J Clin Nurs 17:884-890. doi: 10.1111/j.1365-2702.2007.02054.x

38. Adamson SJ, Sellman JD (2003) A prototype screening instrument for cannabis use disorder: the Cannabis Use Disorders Identification Test (CUDIT) in an alcohol-dependent clinical sample. Drug Alcohol Rev 22:309-315. doi: 10.1080/0959523031000154454

39. Annaheim B, Scotto TJ, Gmel G (2010) Revising the Cannabis Use Disorders Identification Test (CUDIT) by means of Item Response Theory. Int J Methods Psychiatr Res 19:142-55. doi: 10.1002/mpr.308

40. Galéra C, Bouvard MP, Melchior M, et al. (2010) Disruptive symptoms in childhood and adolescence and early initiation of tobacco and cannabis use: The Gazel Youth study. Eur Psychiatry 25:402-408. doi: 10.1016/j.eurpsy.2010.06.002

41. Estévez N, Eich-Höchli D, Dey M, et al. (2014) Prevalence of and associated factors for adult attention deficit hyperactivity disorder in young Swiss men. PLoS One. doi: 10.1371/journal.pone.0089298

42. Molina BSG, Pelham WE (2003) Childhood predictors of adolescent substance use in a longitudinal study of children with ADHD. J Abnorm Psychol 112:497-507.

43. Pingault J-B, Côté SM, Galéra C, et al. (2012) Childhood trajectories of inattention, hyperactivity and oppositional behaviors and prediction of substance abuse/dependence: a 15-year longitudinal populationbased study. Mol Psychiatry 806-812. doi: 10.1038/mp.2012.87

44. Hu M-C, Davies M, Kandel DB (2006) Epidemiology and correlates of daily smoking and nicotine dependence among young adults in the United States. Am J Public Health 96:299-308. doi: 10.2105/AJPH.2004.057232

45. Dey M, Gmel G, Studer J, Mohler-Kuo M (2014) Health-risk behaviors and quality of life among young men. Qual Life Res 23:1009-1017. doi: 10.1007/s11136-013-0524-4

46. Moura HF, Faller S, Benzano D, et al. (2013) The Effects of ADHD in Adult Substance Abusers. J Addict Dis 32:252-262. doi: 10.1080/10550887.2013.824359

47. Kokotailo P (1995) Physical health problems associated with adolescent substance abuse. NIDA Res Monogr 156:112-29.

48. Perkins HW (2002) Surveying the damage: a review of research on consequences of alcohol misuse in college 
populations. J Stud Alcohol, Suppl 91-100. doi: 10.15288/jsas.2002.s14.91

49. Chaiton MO, Cohen JE, O’Loughlin J, Rehm J (2009) A systematic review of longitudinal studies on the association between depression and smoking in adolescents. BMC Public Health 9:356. doi: 10.1186/14712458-9-356

50. Degenhardt L, Hall W, Lynskey M (2003) Exploring the association between cannabis use and depression. Addiction 98:1493-504.

51. Levy S, Katusic SK, Colligan RC, et al. (2014) Childhood ADHD and Risk for Substance Dependence in Adulthood: A Longitudinal, Population-Based Study. PLoS One 9:e105640. doi: 10.1371/journal.pone.0105640

52. Wilens TE (2007) The nature of the relationship between attention-deficit/hyperactivity disorder and substance use. J Clin Psychiatry 68 Suppl 1:4-8.

53. Wilson JJ (2007) ADHD and substance use disorders: developmental aspects and the impact of stimulant treatment. Am J Addict 16 Suppl 1:5-11-13. doi: 10.1080/10550490601082734

54. Biederman J, Petty CR, Woodworth KY, et al. (2012) Adult outcome of attention-deficit/hyperactivity disorder: A controlled 16-year follow-up study. J Clin Psychiatry 73:941-950. doi: 10.4088/JCP.11m07529

55. Wilens TE, Adamson J, Sgambati S, et al. (2007) Do individuals with ADHD self-medicate with cigarettes and substances of abuse? Results from a controlled family study of ADHD. Am J Addict 16 Suppl 1:14-2123. doi: $10.1080 / 10550490601082742$

56. Frei a, Hornung R, Eich D (2010) [Tobacco consumption of adults diagnosed with ADHD]. Nervenarzt 81:860-866. doi: 10.1007/s00115-009-2922-y

57. Loflin M, Earleywine M, De Leo J, Hobkirk A (2014) Subtypes of attention deficit-hyperactivity disorder (ADHD) and cannabis use. Subst Use Misuse 49:427-34. doi: 10.3109/10826084.2013.841251

58. Elkins IJ, McGue M, Iacono WG (2007) Prospective effects of attention-deficit/hyperactivity disorder, conduct disorder, and sex on adolescent substance use and abuse. Arch Gen Psychiatry 64:1145-1152. doi: 10.1001/archpsyc.64.10.1145

59. Bidwell LC, Henry E a., Willcutt EG, et al. (2014) Childhood and current ADHD symptom dimensions are associated with more severe cannabis outcomes in college students. Drug Alcohol Depend 135:88-94. doi: 10.1016/j.drugalcdep.2013.11.013

60. Ameringer KJ, Leventhal AM (2013) Associations between attention deficit hyperactivity disorder symptom domains and DSM-IV lifetime substance dependence. Am J Addict 22:23-32. doi: 10.1111/j.15210391.2013.00325.x 
61. Faraone S V, Biederman J (2005) What is the prevalence of adult ADHD? Results of a population screen of 966 adults. J Atten Disord 9:384-391. doi: 10.1177/1087054705281478

62. Bitter I, Simon V, Bálint S, et al. (2010) How do different diagnostic criteria, age and gender affect the prevalence of attention deficit hyperactivity disorder in adults? An epidemiological study in a Hungarian community sample. Eur Arch Psychiatry Clin Neurosci 260:287-296. doi: 10.1007/s00406-009-0076-3

63. Lee C-T, Clark TT, Kollins SH, et al. (2015) Attention Deficit Hyperactivity Disorder symptoms and smoking trajectories: Race and gender differences. Drug Alcohol Depend 148:180-187. doi: 10.1016/j.drugalcdep.2015.01.002 
Table 1 Socio-demographic characteristics and co-morbid variables according to adult ADHD.

\begin{tabular}{|c|c|c|c|c|c|c|c|}
\hline & & $\begin{array}{l}\text { Participan } \\
\text { without AI }\end{array}$ & & $\begin{array}{l}\text { Participants } \\
\text { with ADHD }\end{array}$ & & & \\
\hline & Total $^{\mathbf{a}}$ & $\mathrm{n}$ & $\%$ & $\mathrm{n}$ & $\%$ & $X^{2}$ & $\mathrm{p}$ \\
\hline \multicolumn{8}{|l|}{ Socio-demographics } \\
\hline$A g e^{b}$ & 4975 & & & & & & \\
\hline$<21$ & & 2321 & 48.6 & 80 & 39.8 & 6.00 & 0.014 \\
\hline$>=21$ & & 2453 & 51.4 & 121 & 60.2 & & \\
\hline Linguistic region $^{b}$ & 4975 & & & & & & \\
\hline German & & 2246 & 47.0 & 62 & 30.8 & 20.36 & 0.000 \\
\hline French & & 2528 & 53.0 & 139 & 69.2 & & \\
\hline Education $^{b}$ & 4975 & & & & & & \\
\hline primary school & & 387 & 8.1 & 30 & 14.9 & 18.65 & 0.000 \\
\hline secondary vocational school & & 2161 & 45.3 & 66 & 32.8 & & \\
\hline high school/university & & 2226 & 46.6 & 105 & 52.2 & & \\
\hline Financial autonomy $^{b}$ & 4975 & & & & & & \\
\hline financial autonomy & & 1802 & 37.7 & 45 & 22.4 & 21.51 & 0.000 \\
\hline partial financial dependency & & 1885 & 39.5 & 91 & 45.3 & & \\
\hline financial dependency & & 1087 & 22.8 & 65 & 32.3 & & \\
\hline Mother's education ${ }^{c}$ & 4975 & & & & & & \\
\hline primary school & & 594 & 12.4 & 32 & 15.9 & 14.85 & 0.001 \\
\hline secondary vocational school & & 3033 & 63.5 & 101 & 50.2 & & \\
\hline high school/university & & 1147 & 24.0 & 68 & 33.8 & & \\
\hline Family affluence ${ }^{c}$ & 4975 & & & & & & \\
\hline above average & & 2169 & 45.4 & 81 & 40.3 & 3.40 & 0.182 \\
\hline average & & 1942 & 40.7 & 84 & 41.8 & & \\
\hline below average & & 663 & 13.9 & 36 & 17.9 & & \\
\hline \multicolumn{8}{|l|}{ Co-morbid disorders } \\
\hline$A S P D^{c}$ & 4975 & & & & & & \\
\hline no & & 4049 & 84.8 & 131 & 65.2 & 55.41 & 0.000 \\
\hline yes & & 725 & 15.2 & 70 & 34.8 & & \\
\hline$M D^{b}$ & 4975 & & & & & & \\
\hline no & & 4651 & 97.4 & 178 & 88.6 & 53.23 & 0.000 \\
\hline yes & & 123 & 2.6 & 23 & 11.4 & & \\
\hline
\end{tabular}

ADHD: Attention-deficit/hyperactivity disorder; ASPD: Anti-social personality disorder; MD: Major depression;

a Total number of participants (n) recorded for this variable; ${ }^{\mathrm{b}}$ Variable was assessed at follow-up; ${ }^{\mathrm{c}}$ Variable was assessed at baseline. 
Table 2 Substance use outcomes according to adult ADHD.

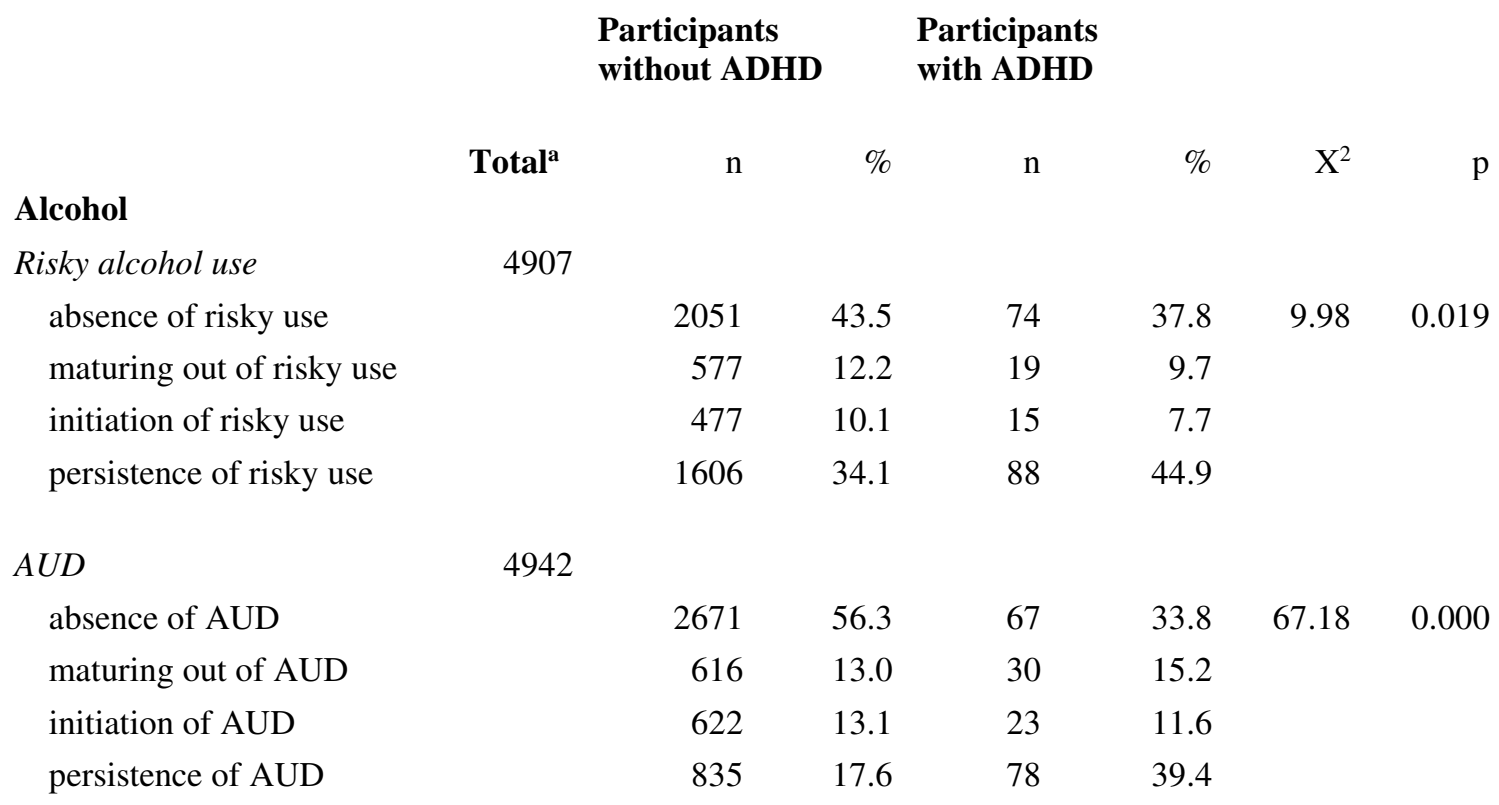

\section{Nicotine}

Risky nicotine use

absence of risky use

maturing out of risky use

initiation of risky use

persistence of risky use

4968

NUD

absence of NUD

maturing out of NUD

initiation of NUD

persistence of NUD

\section{Cannabis}

Risky cannabis use
absence of risky use
maturing out of risky use
initiation of risky use
persistence of risky use
CUD
absence of CUD
maturing out CUD
initiation of CUD
persistence of CUD

4975

$\begin{array}{rrrrrr}3672 & 77.0 & 131 & 65.2 & 20.07 & 0.000 \\ 138 & 2.9 & 7 & 3.5 & & \\ 244 & 5.1 & 10 & 5.0 & & \\ 713 & 15.0 & 53 & 26.4 & & \end{array}$

4531

$\begin{array}{rrrrrr}3759 & 86.7 & 148 & 76.3 & 21.66 & 0.000 \\ 137 & 3.2 & 13 & 6.7 & & \\ 168 & 3.9 & 8 & 4.1 & & \\ 273 & 6.3 & 25 & 12.9 & & \end{array}$

ADHD: Attention-deficit/hyperactivity disorder; AUD: Alcohol use disorder; NUD: Nicotine use disorder; CUD: Cannabis use disorder;

a Total number of participants ( $\mathrm{n}$ ) recorded for this variable, $\mathrm{n}$ varies slightly between variables due to missing data. 
Table 3 Logistic regression analyses with risky use of alcohol, nicotine and cannabis and related SUDs as outcome.

\begin{tabular}{|c|c|c|c|c|c|c|}
\hline & \multicolumn{2}{|l|}{ Risky alcohol use $^{b}$} & \multicolumn{2}{|c|}{ Risky nicotine use $\mathrm{e}^{\mathrm{b}}$} & \multicolumn{2}{|c|}{ Risky cannabis use $^{b}$} \\
\hline & $\mathrm{AOR}^{\mathrm{c}}[\mathrm{CI}]$ & $\mathrm{AOR}^{\mathrm{d}}[\mathrm{CI}]$ & $\mathrm{AOR}^{\mathrm{c}}[\mathrm{CI}]$ & $\mathrm{AOR}^{\mathrm{d}}[\mathrm{CI}]$ & $\mathrm{AOR}^{\mathrm{c}}[\mathrm{CI}]$ & $\mathrm{AOR}^{\mathrm{d}}[\mathrm{CI}]$ \\
\hline \multicolumn{7}{|c|}{$A D H D^{a}$} \\
\hline no & 1.00 & 1.00 & 1.00 & 1.00 & 1.00 & 1.00 \\
\hline \multirow[t]{3}{*}{ yes } & $1.24[0.88,1.75]$ & $1.22[0.86,1.74]$ & $1.27[0.78,2.06]$ & $1.16[0.69,1.94]$ & $1.00[0.55,1.83]$ & $0.75[0.39,1.42]$ \\
\hline & $\mathbf{A U D}^{\mathbf{b}}$ & & $\mathbf{N U D}^{\mathbf{b}}$ & & $\mathbf{C U D}^{\mathbf{b}}$ & \\
\hline & $\operatorname{AOR}^{\mathrm{c}}[\mathrm{CI}]$ & $\mathrm{AOR}^{\mathrm{d}}[\mathrm{CI}]$ & $\mathrm{AOR}^{\mathrm{c}}[\mathrm{CI}]$ & $\mathrm{AOR}^{\mathrm{d}}[\mathrm{CI}]$ & $\mathrm{AOR}^{\mathrm{c}}[\mathrm{CI}]$ & $\mathrm{AOR}^{\mathrm{d}}[\mathrm{CI}]$ \\
\hline \multicolumn{7}{|c|}{$A D H D^{a}$} \\
\hline no & 1.00 & 1.00 & 1.00 & 1.00 & 1.00 & 1.00 \\
\hline yes & $1.70[1.25,2.33]^{* * *}$ & $1.53[1.11,2.12]^{* *}$ & $1.07[0.64,1.81]$ & $0.90[0.51,1.57]$ & $0.73[0.41,1.31]$ & $0.52[0.28,0.98]^{*}$ \\
\hline
\end{tabular}

ADHD: Attention-deficit/hyperactivity disorder; AUD: Alcohol use disorder; NUD: Nicotine use disorder; CUD: Cannabis use disorder;

$* \mathrm{p}<.05, * * \mathrm{p}<.01, * * * \mathrm{p}<.001$;

OR: Odds ratio; CI: $95 \%$ confidence interval;

${ }^{\mathrm{a}}$ Variable was assessed at baseline; ${ }^{\mathrm{b}}$ Variable was assessed at follow-up; ${ }^{\mathrm{c}}$ Adjusted for outcome of interest at baseline; ${ }^{\mathrm{d}}$ Adjusted for outcome of interest at baseline, socio-demographic variables and co-morbid disorders. 
Table 4 Multinomial regression analyses with risky use of alcohol, nicotine and cannabis and related SUDs as outcome.

Risky alcohol use

\begin{tabular}{|c|c|c|c|c|c|c|}
\hline & \multicolumn{2}{|l|}{ maturing out ${ }^{\mathrm{a}}$} & \multicolumn{2}{|l|}{ initiation $^{\mathrm{a}}$} & \multicolumn{2}{|l|}{ persistence $^{\mathrm{a}}$} \\
\hline & Crude OR [CI] & $\mathrm{AOR}^{\mathrm{b}}[\mathrm{CI}]$ & Crude OR [CI] & $\mathrm{AOR}^{\mathrm{b}}[\mathrm{CI}]$ & Crude OR [CI] & $\mathrm{AOR}^{\mathrm{b}}[\mathrm{CI}]$ \\
\hline \multicolumn{7}{|c|}{ ADHD } \\
\hline no & 1.00 & 1.00 & 1.00 & 1.00 & 1.00 & 1.00 \\
\hline yes & $0.91[0.55,1.52]$ & $0.96[0.57,1.62]$ & $0.87[0.50,1.53]$ & $0.93[0.52,1.64]$ & $1.52[1.11,2.08]^{* *}$ & $1.45[1.04,2.02]^{*}$ \\
\hline
\end{tabular}

Risky nicotine use

maturing out

initiation $^{\mathrm{a}}$

persistence $^{\mathrm{a}}$

Crude OR $[\mathrm{CI}]$

$\mathrm{AOR}^{\mathrm{b}}[\mathrm{CI}]$

Crude OR [CI]

Crude OR [CI]

$\mathrm{AOR}^{\mathrm{b}}[\mathrm{CI}]$

\section{ADHD}

no

1.00

yes

$1.42[0.65,3.10]$

1.00

1.00

$\mathrm{AOR}^{\mathrm{b}}[\mathrm{CI}]$

1.00

1.00

$1.15[0.60,2.21]$

$1.00[0.51,1.97]$

$2.08[1.50,2.90] * * *$

$1.75[1.22,2.51]^{* *}$

Risky cannabis use

\begin{tabular}{|c|c|c|c|c|c|c|}
\hline & \multicolumn{2}{|l|}{ maturing out $^{\mathrm{a}}$} & \multicolumn{2}{|l|}{ initiation $^{\mathrm{a}}$} & \multicolumn{2}{|l|}{ persistence $^{\mathrm{a}}$} \\
\hline & Crude OR [CI] & $\mathrm{AOR}^{\mathrm{b}}[\mathrm{CI}]$ & Crude OR [CI] & $\mathrm{AOR}^{\mathrm{b}}[\mathrm{CI}]$ & Crude OR [CI] & $\mathrm{AOR}^{\mathrm{b}}[\mathrm{CI}]$ \\
\hline \multicolumn{7}{|c|}{ ADHD } \\
\hline no & 1.00 & 1.00 & 1.00 & 1.00 & 1.00 & 1.00 \\
\hline yes & $3.43[1.96,6.01]^{* * *}$ & $2.71[1.50,4.92] * * *$ & $2.12[0.97,4.64] \dagger$ & $1.62[0.72,3.65]$ & $2.17[1.35,3.47]^{* * *}$ & $1.33[0.80,2.21]$ \\
\hline
\end{tabular}




\section{AUD}

\begin{tabular}{|c|c|c|c|c|c|c|}
\hline & \multicolumn{2}{|l|}{ maturing out ${ }^{\mathrm{a}}$} & \multicolumn{2}{|l|}{ initiation $^{\mathrm{a}}$} & \multicolumn{2}{|l|}{ persistence $^{\mathrm{a}}$} \\
\hline & Crude OR [CI] & $\mathrm{AOR}^{\mathrm{b}}[\mathrm{CI}]$ & Crude OR [CI] & $\mathrm{AOR}^{\mathrm{b}}[\mathrm{CI}]$ & Crude OR [CI] & $\mathrm{AOR}^{\mathrm{b}}[\mathrm{CI}]$ \\
\hline \multicolumn{7}{|c|}{ ADHD } \\
\hline no & 1.00 & 1.00 & 1.00 & 1.00 & 1.00 & 1.00 \\
\hline yes & $1.94[1.25,3.01] * *$ & $1.76[1.12,2.77]^{*}$ & $1.47[0.91,2.39]$ & $1.32[0.81,2.16]$ & $3.72[2.66,5.21]^{* * *}$ & $3.10[2.16,4.45]^{* * *}$ \\
\hline
\end{tabular}

\section{NUD}

$\begin{array}{ll}\text { maturing out }^{\mathrm{a}} & \text { Crude OR }[\mathrm{CI}]_{\text {ADHD }}\end{array}$

initiation $^{\mathrm{a}}$

persistence $^{\mathrm{a}}$

ADHD

\begin{tabular}{|c|c|c|c|c|c|c|}
\hline no & 1.00 & 1.00 & 1.00 & 1.00 & 1.00 & 1.00 \\
\hline yes & $2.41[1.33,4.36]^{* *}$ & $2.27[1.21,4.24]^{*}$ & $1.21[0.58,2.50]$ & $1.08[0.51,2.29]$ & $2.33[1.50,3.62]^{* * *}$ & $1.97[1.21,3.20]^{* *}$ \\
\hline
\end{tabular}

CUD

\begin{tabular}{|c|c|c|c|c|c|c|}
\hline & \multicolumn{2}{|l|}{ maturing out ${ }^{\mathrm{a}}$} & \multicolumn{2}{|l|}{ initiation $^{\mathrm{a}}$} & \multicolumn{2}{|l|}{ persistence $^{\mathrm{a}}$} \\
\hline & Crude OR [CI] & $\mathrm{AOR}^{\mathrm{b}}[\mathrm{CI}]$ & Crude OR [CI] & $\mathrm{AOR}^{\mathrm{b}}[\mathrm{CI}]$ & Crude OR [CI] & $\mathrm{AOR}^{\mathrm{b}}[\mathrm{CI}]$ \\
\hline \multicolumn{7}{|c|}{ ADHD } \\
\hline no & 1.00 & 1.00 & 1.00 & 1.00 & 1.00 & 1.00 \\
\hline yes & $4.91[2.90,8.30]^{* * *}$ & $3.65[2.08,6.42]^{* * *}$ & $1.29[0.52,3.21]$ & $1.00[0.39,2.55]$ & $2.81[1.76,4.49]^{* * *}$ & $1.69[1.02,2.82]^{*}$ \\
\hline
\end{tabular}

ADHD: Attention-deficit/hyperactivity disorder; AUD: Alcohol use disorder; NUD: Nicotine use disorder; CUD: Cannabis use disorder;

tp $<.10, * \mathrm{p}<.05, * * \mathrm{p}<.01, * * * \mathrm{p}<.001$

OR: Odds ratio; CI: $95 \%$ confidence interval;

${ }^{\text {a }}$ Reference category: absence of the outcome of interest (e.g., absence of AUD); ${ }^{\text {b }}$ Adjusted for socio-demographic variables and co-morbid disorders. 